Pensamiento Crítico No. 7, pág. 135 - 156

\title{
La Neutralidad del Dinero y la Dicotomía Clásica en la Ciencia Económica
}

\author{
Dr. Abraham llanos Marcos
}

Resumen:Neutralidad del dinero y "dicotomía clásica" son temas académicos que constituye a su vez el fundamento de la política monetaria en la mayoría de los Bancos Centrales. El presente artículo, como avance de una investigación en desarrollo, expone los distintos conceptos que sobre el tema sustentan diversas escuelas del pensamiento económico y lo contrasta con el pensamiento crítico que sobre el dinero tiene la corriente marxista, concluyendo preliminarmente que se trata de un ejercicio académico formalmente coherente y blindado muchas veces con una "formalización" matemática pero que es, en esencia, una concepción ideológica defensora de los intereses del capitalismo en crisis.

Palabras claves: dicotomía clásica, neutralidad, dinero, valor de uso, valor de cambio, mercancía, fetichización de las relaciones económicas.

\section{INTRODUCCION}

La "neutralidad del dinero", como concepto académico, aparece en años posteriores a la Primera Guerra Mundial mediante publicaciones de economistas alemanes y holandeses. Sin embargo es Hayek quien introduce este concepto desde la London School of Economics mediante su obra Prices and Production (1931). 
Actualmente en la mayoría de los países el objetivo primordial de los Bancos Centrales es mantener la estabilidad de los precios mediante políticas monetarias de corte restrictivo. Es lo que sucede en nuestro país: la política monetaria que implementa el Banco Central tiene como objetivo fundamental el control de la inflación, sin importar que esto desaliente la inversión productiva y la generación de empleo. Implícitamente las autoridades monetarias asumen que el dinero es neutral, lo que les permite argumentar que su responsabilidad se remite únicamente al control de los precios. Pero, ¿bajo qué condiciones se verifica la neutralidad del dinero? ¿Cuál o cuáles son los conceptos que manejan sobre el dinero?.

En el presente artículo, inicialmente analizaremos y comprobaremos que los supuestos requeridos para que el dinero sea neutral son altamente restrictivos, lo que convierte este concepto en un interesante ejercicio teórico que intenta ocultar el verdadero significado del dinero, $y$, finalmente abordaremos un concepto de dinero que nos permitirá, en próximos ensayos, desarrollar una investigación de mayor alcance sobre este tema en el actual periodo del capitalismo. Este documento expone el avance de una investigación que se viene desarrollando y que debe ser concluida a fines del presente año.

\section{EL PENSAMIENTO ECONÓMICO Y EL DINERO}

Los efectos del dinero sobre la actividad real ha sido motivo de continua controversia entre las diversas escuelas de pensamiento económico (clásica, keynesiana, monetarista, marxista, nueva clásica, real business cycles o RBC, nueva keynesiana, poskeynesiana y austriaca). Para la mayoría de académicos contemporáneos, el dinero se presenta como parte fundamental del sistema económico, pero sus efectos y el papel que desempeña son diferentes para cada una de ellas, más aun en el actual período tardío del capitalismo donde sus contradicciones se exponen y se manifiestan de manera tan evidente. La situación actual de la economía mundial en general, la recesión norteamericana y la depreciación del dólar como moneda universal nos obliga a revisar diversos postulados sobre el rol dinero al interior de la economía capitalista.

\section{ACERCA DE NEUTRALIDAD Y DICOTOMÍA}

En la obra A Modern Guide to Macroeconomics: An Introduction to Competing Schools of Thought los profesores Snowdon, Vane y Wynarczyk (1994) sostienen que para analizar el comportamiento de las variables nominales y reales en un modelo macroeconómico clásico, ${ }^{2}$ se deben tener en cuenta los siguientes componentes: i) la teoría clásica de la determinación del empleo y el producto; ii) la ley de Say, y iii) la teoría cuantitativa del dinero. Los dos primeros 
La neutralidad del dinero y la dicotomía clásica en la ciencia económica

determinarían las variables reales en los mercados de bienes y de trabajo, en tanto que el tercero explicaría la determinación de las variables nominales. Por tanto, el modelo clásico es neutral y dicótomo porque las variables reales se determinan independientemente de las nominales.

Sargent $(1987,46)$ define la neutralidad como: Un sistema posee la propiedad de neutralidad si se puede escribirse de forma que cada ecuación contenga únicamente magnitudes medidas en unidades 'reales'. Así pues, si dondequiera que aparezca una magnitud medida en dólares (como la cantidad de dinero o bonos o el salario) la dividimos por otra variable igualmente medida en dólares (como el precio o el salario), el modelo describiría relaciones entre magnitudes reales. Si esto es cierto, doblar o triplicar las magnitudes nominales no puede tener efecto alguno sobre los valores de equilibrio de las variables reales.

Y la dicotomía clásica como: Se dice que un modelo macroeconómico dicotomiza si un subconjunto de ecuaciones puede determinar los valores de todas las variables reales, sin que el nivel de oferta monetaria juegue un papel en la determinación de sus valores de equilibrio. Dados los valores de equilibrio de las variables reales, el nivel de la oferta monetaria ayuda a determinar los valores de equilibrio de las variables nominales que sean endógenas, pero no puede influenciar ninguna variable real. En un sistema que dicotomiza, los valores de equilibrio de todas las variables reales son independientes del nivel absoluto de precio. En tal sistema "el dinero es un velo".

Aunque Sargent (1987) expone la diferencia entre neutralidad del dinero y dicotomía clásica, para los fines expositivos del presente documento consideraremos ambos conceptos como iguales.

Snowdon, Vane y Wynarczyk revisan el papel de la moneda en cada escuela sin diferenciar ninguno de los dos conceptos: el concepto de neutralidad aparece mientras que el de dicotomía no. Este último sólo se menciona explícitamente cuando se discute neutralidad en el contexto del modelo clásico y de los nuevos keynesianos. Pero en el resto de escuelas, la exposición sobre la moneda y sus efectos en la economía, hace uso del concepto de neutralidad.

De otro lado, para Wicksell el equilibrio monetario implica que se verifiquen en forma simultánea una serie de condiciones. Hayek plantea la neutralidad en términos de los efectos que generan las variaciones monetarias sobre el nivel de los precios relativos. Patinkin incorpora el concepto de neutralidad del dinero de Hayek al marco de la síntesis neoclásica y establece una serie de hipótesis bajo la cuales el dinero puede ser considerado neutral.

El proceso acumulativo de Wicksell es una aportación importante para explicar la inestabilidad en el nivel agregado de los precios donde termina aceptando la dicotomía clásica y las conclusiones de la teoría cuantitativa. Mientras que Hayek critica dicha teoría e invalida a la dicotomía clásica, lo que le sirve de base para desarrollar el concepto de neutralidad del dinero. Patinkin comparte con Hayek el hecho de que también invalida dicha dicotomía, pero señala que no significa que las conclusiones de la teoría cuantitativa dejen de ser válidas. Estas 
autores hay un esfuerzo para intentar integrar la teoría monetaria y la teoría del valor.

Wicksell, al igual que otros teóricos cuantitativos, consideran implícitamente que el objetivo de la política monetaria es mantener la estabilidad en el valor del dinero. Este planteamiento es relativamente frágil porque se deduce analizando un caso particular de su teoría y suponiendo que el Banco Central está en manos del sector público.

Hayek considera que en la definición de neutralidad del dinero el nivel promedio de precios no juega ningún papel, por lo tanto, lo excluye de su análisis. Sin embargo, los cambios en el nivel agregado de precios, generan procesos de redistribución del ingreso que a su vez producen modificaciones en la composición de los patrones de compra, alterando los precios relativos. La redistribución del ingreso no genera efectos en las variables reales únicamente bajo supuestos muy restrictivos, por lo tanto, la definición de neutralidad del dinero que el autor propone es incoherente.

Antes de la publicación de Prices and Production, las ideas dominantes en materia de teoría monetaria eran las planteadas por la teoría cuantitativa, el dinero a fin de cuentas constituía solo un velo, lo que determinaba el funcionamiento del sistema económico eran las variables reales. Estas ideas eran propuestas desde una perspectiva agregada, un antecedente importante en el desarrollo posterior de la teoría de Keynes, la cual dio origen a la teoría macroeconómica propiamente dicha.

Hayek introduce los fundamentos microeconómicos en el análisis del papel del dinero en el sistema económico porque las relaciones que se establecen entre variables agregadas son incapaces de explicar el comportamiento de los agentes económicos individuales; el sistema económico es mucho más rico y complejo que el planteamiento mecanicista de la teoría cuantitativa.

Pantinkin influenciado históricamente por Wicksell y Hayek asume la neutralidad del dinero aunque reflejando las ideas de autores como Modigliani (1944), uno de los arquitectos de la síntesis neoclásica. Y es que la no neutralidad del dinero es la característica principal del modelo IS-LM, en el que se asume rigidez salarial.

Por último, según Cagan $(1969,207)$, "el dinero puede ser definido como neutral si cambios en la oferta no tienen efectos sobre los gastos reales y la asignación de recursos".

\section{LA NEUTRALIDAD DEL DINERO EN EL CORTO PLAZO}


a) Para los Clásicos, se obtiene cuando ante cualquier cambio en la política monetaria, las variables reales no reaccionan, y únicamente se ven afectadas por cambios en otras variables reales. Para que esto suceda (ver Gráfico 1) la oferta agregada debe ser perfectamente inelástica ante cualquier perturbación originada en la demanda agregada: en una expansión monetaria, los precios absorben completamente el shock (el nivel de precios pasa de P0 a P1) y el PIB se mantiene en su tasa natural (Yn).

\section{Gráfico 1 Modelo Clásico}

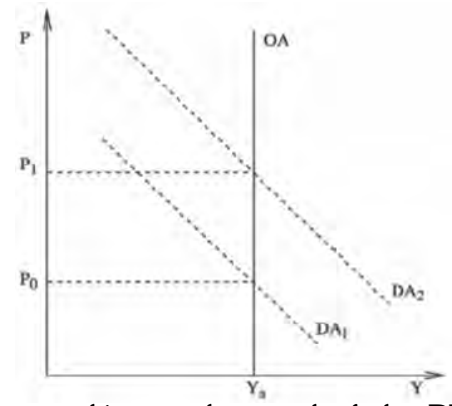

Bajo este esquema, la escuela clásica, la de los nuevos clásicos y la escuela de los RBC, defienden la dicotomía clásica.

Para la escuela clásica el producto está determinado por la estructura del mercado laboral y de bienes, y en ella el dinero entra como un medio de cambio. Esta formalización se encuentra en la Teoría Cuantitativa del Dinero (ecuación 1).

M: oferta monetaria

$$
\mathrm{P}=\mathrm{M} * \mathrm{~V} * \mathrm{Y}
$$

$\mathrm{V}$ : velocidad del dinero

P: nivel de precios

Y: producto real, el cual está fijo (determinado por la teoría clásica de la determinación del producto y el empleo y la ley de Say).

De la ecuación 1 se tiene que: $\quad \pi=\frac{\mathrm{dM} / \mathrm{dt}}{\mathrm{M}}+\frac{\mathrm{dV} / \mathrm{dt}}{\mathrm{V}}+\frac{\mathrm{dY} / \mathrm{dt}}{\mathrm{Y}}$

$\pi=$ inflación

$\frac{\mathrm{dM} / \mathrm{dt}}{\mathrm{M}}$ Tasa de crecimiento de la oferta monetaria

$\frac{\mathrm{dV} / \mathrm{dt}}{\mathrm{V}}$ Tasa de crecimiento de la velocidad del dinero

$\frac{\mathrm{dY} / \mathrm{dt}}{\mathrm{Y}}$ Tasa de crecimiento de la economía

Para los clásicos la velocidad del dinero tiende a ser constante, por lo que (dV/dt)/V = 0, y el producto se encuentra en su nivel natural o de pleno empleo, $(\mathrm{dY} / \mathrm{dt}) / \mathrm{Y}=0$. 
Por tanto, de la ecuación 2 se tiene que: $\quad \pi=\frac{d M / d t}{M}$

Es decir, la inflación está enteramente explicada por el crecimiento de la oferta monetaria.

En la gráfica 1: la interacción de la oferta agregada, OA, con la demanda agregada, DA, determinan el nivel de precios de equilibrio a un nivel de producto de pleno empleo, y cualquier perturbación en la demanda afectará únicamente al nivel de precios, es decir a la inflación.

\section{b) Para los Nuevos Clásicos}

La condición de neutralidad requiere ${ }^{6}$ (ver Snowdon, Vane y Wynarczyk, cap. 5, 1994) que se cumpla las perturbaciones en la DA deben ser anticipadas y/o anunciadas. De esta manera, los agentes se acomodan a la política monetaria anticipando cualquier cambio en ella. En esta situación, rige una economía de tipo clásica y la condición de neutralidad del dinero se cumple plenamente.

Sin embargo, si la perturbación no es anunciada o no esperada, el dinero sí puede tener efectos reales, violando la neutralidad, pero sólo en el corto plazo $(\mathrm{CP})$ : si los agentes esperan una inflación y forman sus expectativas, la autoridad monetaria puede generar una sorpresa monetaria ${ }^{7}$ y obtener ganancias temporales en empleo y producto $^{8}$, dado que la economía retorna a su nivel de equilibrio de largo plazo (LP) con un mayor nivel de precios (ver Gráfico 2), donde se observa que la economía hace el tránsito del punto A al B y luego al C?

Modelo Nuevo Clásico con

Sorpresas Monetarias.

(Gráfico 2)

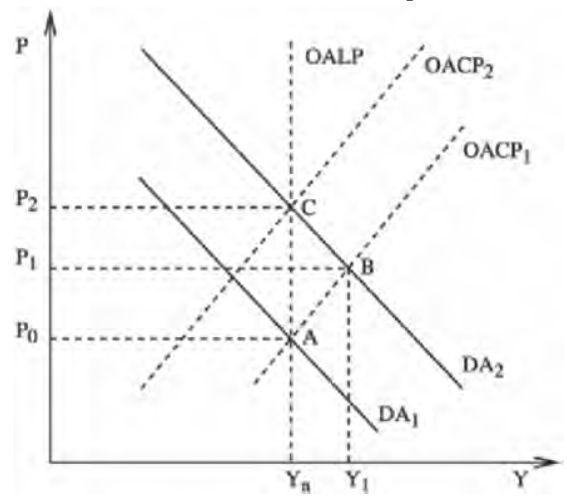

La escuela de los nuevos clásicos supone que los agentes poseen expectativas racionales, es decir, conocimiento acerca del comportamiento de la economía y de las decisiones de las autoridades económicas.

Para que esos cambios anunciados y anticipados en la política monetaria no tengan efectos reales deben darse dos situaciones: 
La neutralidad del dinero y la dicotomía clásica en la ciencia económica

i) Que la autoridad encargada del manejo monetario posea credibilidad ante los agentes.

ii) Que esos cambios anunciados sean efectivamente realizados y no se pretenda engañar a los agentes, es decir, que no deben existir sorpresas monetarias.

La primera condición es bastante importante para la autoridad monetaria ya que, si posee credibilidad, los agentes incorporarán a sus expectativas los cambios anunciados en la oferta monetaria, reduciendo a cero los costos de la desinflación.10 En la segunda condición, si la autoridad monetaria anuncia metas monetarias que realmente va a cumplir, los agentes incorporan el anuncio a sus expectativas y el encargado de la política cumple su anuncio para evitar violar la primera condición.11

Por lo expuesto la inconsistencia dinámica previene a los agentes de utilizar sorpresas monetarias para tener ganancias en el producto, debido a que si en el futuro se implementa un programa de estabilización de la inflación, la razón de sacrificio puede ser positiva, con amplios costos sobre el producto. Así, la neutralidad desaparece en el CP cuando la autoridad monetaria pretende explotar el tradeoff entre inflación y desempleo y los agentes con expectativas racionales incorporan ese comportamiento en su conjunto de información.

En conclusión, un shock de oferta monetaria puede tener efectos diferentes que dependen de la credibilidad en la autoridad monetaria y de si ha sido anunciado o no. Si el shock es completamente sorpresivo, los agentes se enfrentan al problema de extracción de señales y no saben si el aumento en los precios es un aumento en el nivel general de precios o en su precio relativo. Si la historia muestra que la variabilidad en el nivel de precios es alta, el problema de la extracción de señales es más profundo, y esto puede afectar los planes de la autoridad monetaria si lo que pretende es reducir la inflación.

Debido a la mala percepción de los agentes acerca del cambio en los precios, es posible que los productores cambien su nivel de producción si ellos perciben el cambio en el nivel de precios como un cambio en el precio relativo de su producto. Lo anterior genera una variabilidad en el producto de la economía, que se incrementa entre más inestable sea el nivel de precios. En ausencia de shocks monetarios, para los nuevos clásicos, la economía siempre se encontrará en el producto de equilibrio o su nivel natural. Por lo tanto, la neutralidad del dinero se rompe si existen sorpresas monetarias que confunden a los agentes económicos. En efecto, cambios anunciados y anticipados que sean creíbles permiten aceptar la propiedad de dicotomía en los modelos de los nuevos clásicos. Cualquier ruptura de estas condiciones genera efectos reales en la economía.

A pesar de lo anterior, a los nuevos clásicos se les presenta un problema: ¿Qué tan coherente era su apreciación acerca de la posibilidad de sorpresas monetarias, en un ambiente 
de expectativas racionales? Los desarrollos teóricos relacionados con la inconsistencia dinámica ${ }^{12}$ prácticamente impedían la cohabitación del supuesto de expectativas racionales con la posibilidad de que el Banco Central generara sorpresas monetarias, puesto que a LP las variables reales no se afectaban y se generaba una mayor inflación. Es decir, la posibilidad de explotar el tradeoff entre inflación y desempleo desaparece por la inconsistencia dinámica.

\section{c) Para la corriente del Real Business Cycles o RBC}

Los nuevos macroeconomistas clásicos fueron un paso más allá de sus planteamientos teóricos, surgiendo lo que se conoce como la escuela de los $\mathrm{RBC}^{13}$, que defiende la dicotomía clásica tanto en el CP como en el LP. Para ellos, el dinero no es importante para explicar las fluctuaciones de la economía, ya que la política monetaria responde a cambios en la actividad real. De acuerdo a Snowdon (1994, 253), la correlación positiva entre dinero y producto es un ejemplo de "causación en reversa; esto es, expectativas de expansiones futuras en el producto llevan a expansiones actuales en la oferta monetaria". Así el dinero se convierte en una variable endógena que aunque es controlada por el Banco Central, depende de las expectativas sobre las variables reales para determinar su comportamiento. Por tanto, las fluctuaciones ya no eran generadas o explicadas por la política monetaria, sino por los choques tecnológicos que hacían cambiar las decisiones intertemporales óptimas de los agentes entre ocio y trabajo.

Para los RBC el dinero es endógeno al sistema económico. Partiendo de la ecuación 1, la relación de causalidad no va de derecha a izquierda, sino de izquierda a derecha, es decir el PIB nominal es el que determina la cantidad de dinero de la economía. Así, los cambios planeados en el producto son los que afectan la demanda de dinero, y el dinero resulta siendo una variable endógena. Esta visión es defendida por King y Plosser $(1984)_{14}^{14}$ para quienes la industria financiera es "una proveedora de flujo de servicios de cuenta que ayudan a facilitar las transacciones de mercado". Por lo tanto, la única función del dinero es la de medio de cambio y esa característica no afecta el sector real de la economía. Cualquier cambio en la oferta monetaria es producto del ritmo de la actividad económica real, y no, como se afirma tradicionalmente, que el dinero es una variable exógena. King y Plosser muestran que los movimientos en el mercado de dinero están positivamente relacionados con los movimientos en el producto. Snowdon, Vane y Wynarczyk $(1994,153)$ lo presentan de la siguiente forma: [...] en tal situación el dinero es endógeno y las correlaciones de dinero a producto que observamos son evidencia de una causación en reversa; esto es, expectativas de expansiones futuras en el producto llevan a incrementos corrientes en la oferta de dinero.

Esto garantiza que la dicotomía se cumple para los modelos RBC, ya que no son cambios exógenos en la oferta monetaria los que tienen efectos en la economía, sino que los movimientos en el producto generan cambios endógenos en la cantidad de dinero. Es decir, los movimientos en las variables reales generan cambios en las nominales y no viceversa. 


\section{d) Para Keynes y los Keynesianos}

Keynes reconoce que en el LP la economía tiende a funcionar como lo sugieren los clásicos, ${ }^{15}$ pero este período de tiempo es irrelevante, puesto que en el LP todos estaremos muertos. Así queda definida su no neutralidad del dinero. Para Keynes y los keynesianos ${ }^{16}$ la dicotomía no se presenta, dado que el desempleo es persistente y con sólo políticas de demanda agregada la economía puede alcanzar su nivel de equilibrio. El hecho de que se de espacio para que las políticas monetaria y fiscal ${ }^{17}$ puedan afectar el nivel de ingreso, permite concluir que la neutralidad no se cumple desde el punto de vista de esta escuela.

En el Gráfico 3, vemos que sólo las políticas de impulso a la demanda agregada pueden acercar a la economía a su nivel de LP.

La noción de inestabilidad del sistema capitalista de Keynes y de los keynesianos es la que sustenta la imposibilidad de que la economía alcance su nivel de equilibrio de LP y hace que el desempleo sea persistente, constituyéndose en una situación de equilibrio con desempleo. En conclusión, Keynes rompe con la tradición clásica que sustenta su análisis en la ley de Say, según la cual cualquier crecimiento de la oferta es absorbido por un crecimiento igual en la demanda, para referirse a la insuficiencia en la demanda efectiva como causante principal de las fluctuaciones.

Gráfico 3 Modelo Keynesiano

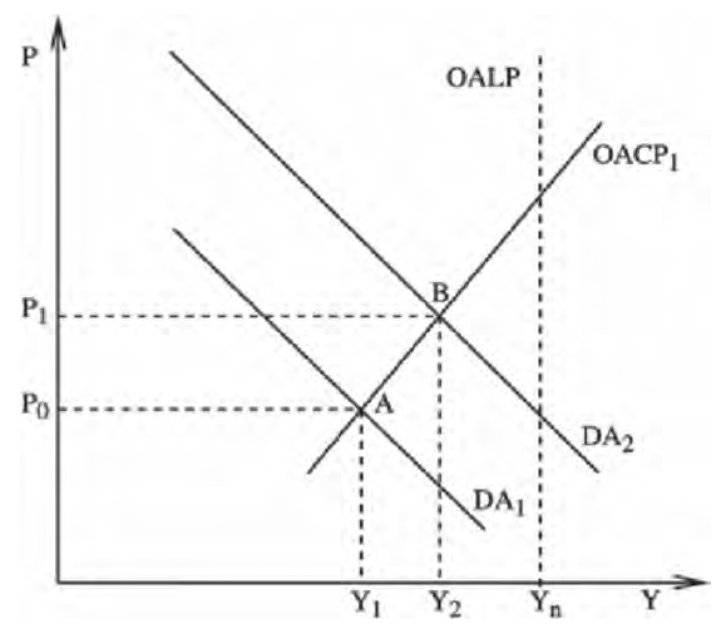

La utilización continua de los instrumentos de política económica podría romper con la persistencia del desempleo. Keynes no consideró de manera explícita la posibilidad de que la economía alcanzara niveles de producto por encima de los de equilibrio. Sin embargo, ante un recalentamiento de la economía, la política económica se puede utilizar para evitar una mayor expansión y guardar reservas ${ }^{18}$ para cuando el ciclo entre en una fase recesiva ${ }^{19}$. 


\section{e) Para la teoría cuantitativa}

En la ecuación 1 tenemos que la causalidad se presenta de izquierda a derecha (a diferencia de los RBC). La política monetaria afecta el PIB nominal y sus efectos se distribuyen en cambios en el nivel de precios y en el nivel de producto real, eliminando la dicotomía desde el punto de vista keynesiano. A diferencia de los clásicos, en donde las perturbaciones son absorbidas enteramente por los precios, la existencia de rigideces impide que los precios se ajusten, por lo que parte de los choques también es absorbido por el PIB real.

\section{f) Para las Nuevas Corrientes}

Los nuevos clásicos reconocen que el análisis keynesiano buscó mejorar los métodos utilizados hasta los años treinta; no obstante, las fallas teóricas y empíricas de la economía keynesiana abrieron nuevas perspectivas a la teoría macroeconomía en los setentas (Lucas y Sargent, 1978 y Mankiw, 1990). Los nuevos keynesianos aparecieron como respuesta a los nuevos clásicos, utilizando las expectativas racionales y el agente representativo para microfundamentar la macroeconomía. Con sus modelos de características keynesianas concluyeron que la economía presenta fallas de coordinación y que el equilibrio de pleno empleo es más distante que el equilibrio con desempleo. ${ }^{20}$

Con la microfundamentación de la macro, aparece la rivalidad más importante en la teoría macroeconómica de los últimos 30 años. Los nuevos keynesianos defienden la no neutralidad, por lo que la política económica ocupa un lugar importante, aunque restringido por la inconsistencia dinámica y por la limitada información que tienen las autoridades para calcular sus intervenciones.

A diferencia de los nuevos clásicos, que suponen que los precios son totalmente flexibles y que los productores son tomadores de precios, los nuevos keynesianos suponen que el ajuste de precios es lento ${ }^{21}$ y que los productores son formadores de precios, ya que predomina el poder de mercado y la existencia de monopolios. Cuando existe flexibilidad de precios, el ajuste de los precios es instantáneo ante un shock en la economía; pero su rigidez hace que el ajuste sea costoso y cause ciclos económicos.

En cuanto a la neutralidad, los nuevos keynesianos afirman que una política monetaria anti-inflacionaria hace que la razón de sacrificio sea positiva, reduciendo la efectividad de la desinflación sin costos para el producto (Mankiw, 2000). Por tal razón, no existe la dicotomía clásica en los modelos de los nuevos keynesianos.

Por otro lado, los poskeynesianos, -aunque tratan el dinero como endógeno- tampoco suponen la neutralidad por los mismos motivos de Keynes. Para ellos, a diferencia de los RBC, el 
sector bancario es un agente activo en la creación de crédito para financiar el gasto planeado. El crédito nace por la existencia del gasto planeado pero, cuando se ejecuta, éste se convierte en gasto corriente o actual permitiendo que el dinero tenga efectos reales en la economía. De esta manera, los poskeynesianos continúan con la tradición keynesiana de un modelo de economía de producción, usando dinero, moviéndose a través del tiempo, sujeto a incertidumbre y a la posibilidad de errores. La neutralidad se viola y se rechaza puesto que la economía es una economía monetaria de la producción, donde el dinero juega un papel activo en la determinación de las variables reales.22

\section{g) Para los Monetaristas}

Suponen la posibilidad de que en el CP la neutralidad del dinero no se cumple, y la Gran de Depresión es para Milton Friedman el mejor ejemplo de que la política monetaria no es neutral. El comportamiento de la Reserva Federal, al facilitar una contracción monetaria, dejó a la economía sin liquidez y produjo la deflación que ocasionó la pronunciada contracción real en los Estados Unidos. ${ }^{23}$ Los hechos presentados en la Gran Depresión llevaron a Friedman a considerar que la política monetaria, cuando se utiliza de manera activa, puede producir fluctuaciones con costos sobre el bienestar. La diferencia fundamental con los keynesianos es el período de ajuste de los precios. Aunque para los monetaristas los precios son flexibles, el proceso de formación adaptativa de las expectativas por parte de los agentes permite que el dinero tenga efectos reales. Dado que ese mecanismo implica tener en cuenta el pasado de las variables, los agentes adaptan sus proyecciones de acuerdo a los hechos del pasado, abriendo la posibilidad de cometer errores sistemáticos, que hacen que el dinero sea un factor importante de las fluctuaciones en la economía.

Con la discusión del poder desestabilizador de la política monetaria, Friedman concluye que lo mejor es que la política monetaria tenga reglas, en lugar de utilizarla de manera activa, ya que la información disponible en el mercado impide alcanzar los objetivos de estabilización propuestos. El principal ejemplo es el de los rezagos endógenos y exógenos ${ }^{24}$ a los que se enfrenta la política económica, que reducen su poder para mitigar los ciclos económicos.

\section{h) Para la Escuela Austriaca}

Al igual que para los keynesianos y sus seguidores, la neutralidad no existe y el dinero afecta los precios relativos y la estructura del tiempo de la producción. Para ellos, no sólo el tamaño de la oferta monetaria sino también el mecanismo de transmisión de la política monetaria en la economía, incide significativamente en las variables reales. Por lo tanto, el dinero puede ser un factor estabilizador o desestabilizador de la economía, y es importante para explicar las fluctuaciones económicas. Según los austriacos, "no sólo el tamaño del cambio en la oferta monetaria sino también la ruta por la cual el dinero entra al sistema económico, afecta las 


\section{Abraham Llanos Marcos}

variables reales y el resultado final del mercado" (Snowdon, Vane y Wynarczyk, 1994, 355). En esta escuela, el dinero siempre importa y, por lo tanto, no es neutral frente a la economía real.

\section{LA NEUTRALIDAD DEL DINERO EN EL LARGO PLAZO}

En la discusión del LP existe un mayor consenso. Para los clásicos, monetaristas, nuevos clásicos y RBC la neutralidad existe, pero no para los nuevos keynesianos y austriacos. No se tienen en cuenta los keynesianos ni poskeynesianos, puesto que el LP no existe y es irrelevante.

En lo que respecta a los clásicos, la dicotomía se cumple en el corto y el largo plazo, ya que el producto siempre será de equilibrio y al estar en su nivel natural no va a cambiar. Afirmar que en el largo plazo el producto es de equilibrio, es decir está en el pleno empleo de los recursos productivos, impide que el dinero tenga efectos reales en la economía. Cualquier shock monetario es absorbido completamente por los precios, generando inflación (el esquema es igual al del Gráfico 1).

Para los monetaristas sí existe la dicotomía a largo plazo. Afirman que debido a la existencia de una curva de Phillips completamente vertical a largo plazo, la economía se encuentra en equilibrio de pleno empleo, situándose en la tasa natural de desempleo. Cualquier cambio en la cantidad de dinero sólo produce situaciones de mayor inflación. Por tal razón, la inflación es un fenómeno monetario.

En cuanto a los nuevos clásicos, aunque la neutralidad no se cumple en el corto plazo (por la existencia de sorpresas monetarias), en el largo plazo se cumple la propiedad de dicotomía. Al igual que los monetaristas, ellos sostienen que un cambio en la cantidad de dinero genera una mayor inflación. Cuando los nuevos clásicos incorporan las expectativas racionales en sus modelos suponen que los agentes, ante cualquier expectativa de cambio en la oferta monetaria, incluyen inmediatamente dichos cambios a sus expectativas, generando una mayor inflación en el corto plazo. Además, el problema de la inconsistencia dinámica, sumado al supuesto de expectativas racionales, impide que la política económica sea utilizada sistemáticamente para afectar el producto ${ }^{25}$ y sólo puede desembocar en procesos inflacionarios. Pero si las autoridades monetarias tienen en cuenta ese problema de inconsistencia dinámica, tanto a corto como a largo plazo, la neutralidad se cumple sin ningún atenuante.

Para la escuela de los RBC, si la neutralidad existe a corto plazo, también se cumple en el largo plazo. La moneda es endógena y simplemente responde a cambios en la actividad económica real. Las fluctuaciones económicas son explicadas por el lado de la oferta agregada y la moneda no tiene ninguna incidencia real. 
Para los nuevos keynesianos y la escuela austriaca, la elección bajo incertidumbre y la existencia del riesgo impiden que el dinero no afecte el mercado real, negando por completo la existencia de la dicotomía. Para los nuevos keynesianos la rigidez de los precios y las imperfecciones del mercado se cumplen tanto a corto como a largo plazo. El dinero siempre va a tener efectos reales, más aun cuando se usa como un instrumento de la demanda agregada para empujar la economía al pleno empleo.

Para los austriacos, si el riesgo y la incertidumbre afectan el corto plazo, estos mismos factores afectan la actividad económica real en el largo plazo, y el dinero siempre va a ser un factor importante para determinar las variables reales.

\section{Marx y el dinero}

El dinero expresa una relación social en la cual el potencial del trabajo social se ha fragmentado en trabajos privados desempeñados independientemente entre sí. Los productores debido a esto, sólo entran en contacto social a través del intercambio de los productos de sus trabajos, estos productos adquieren la forma de mercancía; éstas a su vez, poseen un valor de cambio y la producción generalizada de la mercancía sólo es posible si este valor de cambio las confronta, independientemente, como dinero. ${ }^{26} \mathrm{El}$ dinero, pues, se encuentra en la raíz de la naturaleza social del trabajo privado de los productores de mercancías, como en el hecho de que este carácter social sólo puede prevalecer por la vía indirecta del intercambio de mercancías, el mercado y la apropiación privada del valor producto (en el modo de producción capitalista: apropiación de plusvalía por el capital). "El dinero no es, en realidad, otra cosa que una especial expresión del carácter social del trabajo y de sus productos, la cual, sin embargo, como contraste con la base de la producción privada, tiene necesariamente que aparecer siempre, en última instancia, como un objeto, como una mercancía especial al lado de otras mercancías" ${ }^{27}$

El hecho de que el carácter social del trabajo productor de mercancías no es dado como un dato a priori crea la necesidad de material dinero, en otras palabras, de que el valor se incorpore en el valor de una mercancía específica: un equivalente universal: "La necesidad misma de transformar el producto o la actividad de los individuos ante todo en forma de valor de cambio, en dinero, y de que sólo en esta forma de cosa ellas adquieran y manifiesten su poder social, demuestra dos cosas distintas: 1) que los individuos siguen produciendo sólo para la sociedad y en sociedad; 2) que su producción no es inmediatamente social, no es el fruto de una asociación, que reparte en su propio interior el trabajo". ${ }^{8}$

Marx explicó por qué el "dinero trabajo" que expresaría tan sólo un número específico de horas de trabajo ("valor") no podía funcionar como un equivalente universal para las mercancías en una sociedad productora de mercancías. ${ }^{29}$ De esta manera, superó el dualismo tradicional (todavía presente en David Ricardo) entre la Teoría del Valor-Trabajo que determina el Valor de las mercancías y la Teoría Cuantitativa que determina el "Valor Monetario", pudiendo así desarrollar una Teoría Económica coherente y uniforme sobre la base de la Teoría del 
Valor-Trabajo. "El dinero no nace de una convención, así como tampoco nace de una convención el Estado. Nace naturalmente del cambio y en el cambio, es su producto" 30

Cualquier intento de atribuir la determinación del "Valor Monetario" a alguna otra "convención", compulsión estatal o mero "reflejo de los valores de las mercancías" conduce a contradicciones muy serias. Es el error más evidente de Rudolf Hilferding que en su famoso libro "El Capital Financiero" postula una Teoría de "valor de circulación socialmente necesaria" derivada directamente del producto total del valor de las mercancías (la suma de los valores de todas las mercancías en circulación) ${ }^{31}$ Aún antes de la I Guerra Mundial, Kautsky había analizado el error básico de esta teoría del dinero, aunque no llevó su crítica hasta sus correctas conclusiones. 32 . Lenin usó una sentencia anotada al margen del texto sobre la teoría del dinero elaborada por Hilferding: falsa. ${ }^{33}$ En tiempos reciente la profesora Suzanne de Brunhoff también critica esta Teoría "marxiana" del dinero aunque no menciona el elemento crucial en la Teoría del dinero de Kart Marx, como tampoco lo hizo Kautsky:

"La diferencia entre precio y valor, entre la mercancía medida a través del tiempo de trabajo de la cual es producto, y el producto del tiempo de trabajo por el cual ella se cambia, crea el requerimiento de una tercera mercancía como medida en la que se expresa el valor de cambio real de la mercancía. Dado que el precio no es idéntico al valor, el elemento que determina el valor - el tiempo de trabajo - no puede ser el elemento en que se expresan los precios, ya que el tiempo de trabajo debería expresarse al mismo tiempo como el determinante y lo no-determinante, como lo igual y lo no igual a sí mismo". ${ }^{5}$

La fórmula de Hilferding, "la suma de los valores de todas las mercancías" dividido por la velocidad de la circulación del dinero, carece doblemente de sentido: primero, porque la "suma de los valores de todas las mercancías" representa la suma de cantidades de trabajo no homogéas, que solo pueden ser reducidas a tiempo de trabajo socialmente necesario por medio del intercambio y de proporciones particulares distintas; segundo, porque tal cantidad de trabajo no puede en la realidad ser dividido por la velocidad de circulación del dinero: cinco millones de horas de trabajo dividido entre monedas de oro o billetes de banco que circulan 25 veces al año, es una fórmula vacía.

Este "modo científico tambien lo encontramos en versiones modernas del Monetarismo que formalmente parecen coherentes, respaldadas en la contabilidad social, en la matemática formalmente aplicada y en el fetichismo del dinero, pero que se desmorona en periodos de crisis donde las contradicciones del capital aparecen en toda su dimensión haciendo evidentes las raíces profundas que establece la mercancía con el dinero electrónico que parece tener vida propia y autónoma en la superficie del sistema. Volveremos sobre este tema mas adelante.

Ciertamente, si la "suma de los valores de todas las mercancías" es reemplazada por la "suma de los precios de todas las mercancías", y si aceptamos que el precio es la expresión monetaria (forma monetaria) del valor, entonces la suma de los precios puede verse como una 
relación, a saber, entre el valor cambiante de las mercancías y el valor cambiante de la mercancía dinero, el material dinero.

Marx extrajo de su definición general del dinero la importante conclusión de que las mercancías solo pueden entrar en circulación si previamente se les ha asignado un precio ideal. ${ }^{36}$ El error de Hilferding estaba estrechamente relacionado con el hecho de que no llegó a entender el antagonismo entre el valor de uso y el valor de cambio, lo que le conduce a la hipótesis equivocada de un cartel universal, cuya producción proporcional lo pondría a salvo de las crisis. En el campo popular este error se nota parcialmente en Nikolai Bujarin; pero en la actualidad es todo un "boom literario" a través de los gurues del "management moderno" quienes postulan una economía mundial sin países y liderados por las corporaciones imperialistas "globales".

Cualquier análisis objetivo del problema dinero debe, a nuestro criterio, partir de un análisis entre el valor cambiante de las mercancías y el valor cambiante de la mercancía dinero, del material dinero: "Para poder servir como medida de los valores, el oro tiene que ser virtualmente un valor variable, puesto que solamente como tiempo de trabajo materializado puede el oro convertirse en equivalente de otras mercancías, y porque el mismo tiempo de trabajo se realiza siguiendo la variación de las fuerzas productivas del trabajo real, en volúmenes distintos de los mismos valores de uso" 37

\section{CONCLUSIONES}

El dinero es un tema de debate e investigación. Las diferentes escuelas, partiendo de distintos supuestos, han llegado a diversas conclusiones sobre la participación del dinero y sus efectos en el sistema económico. Este artículo resume el debate de la neutralidad del dinero entre las escuelas más representativas.

En el corto plazo, la neutralidad del dinero se cumple con total perfección en la escuela clásica y RBC. En la primera, el dinero es exógeno al sistema económico y su participación no cambia las variables reales. La economía, al estar en su nivel de pleno empleo, traslada a los precios y a las demás variables nominales los efectos de un choque de la política monetaria.

Para la escuela de los RBC el dinero es endógeno y sólo sirve para proveer de liquidez a los empresarios que desean realizar una inversión. El dinero cambia en la medida en que las variables reales cambian y acompaña sus fluctuaciones. Éstas son producto de choques tecnológicos y de cambios en las decisiones óptimas de los agentes entre ocio y trabajo.

En el campo de la endogeneidad del dinero, Tobin (1970) mostró que incluso allí la neutralidad se puede romper. La idea principal es que la expansión del ingreso está precedida de una expansión en la oferta monetaria, producto de una expansión del crédito del sector financiero al 
sector real de la economía. Los agentes realizan planes de gasto, gasto planeado, y para financiarlo acuden al sector financiero en busca de crédito.

Cuando obtienen la liquidez, el gasto planeado pasa a ser gasto corriente. Así, aunque el dinero depende del gasto planeado, de donde proviene su endogeneidad, afecta las variables reales vía cambios en el gasto corriente (Snowdon, Vane y Wynarczyk, 1994, 253-255).

Aunque la respuesta de los $\mathrm{RBC}$ a los planteamientos de Tobin se refiere a la validez empírica del papel del dinero en las fluctuaciones económicas, el debate entre los monetaristas y los keynesianos acerca de los ciclos muestra que aún la discusión no está cerrada y que el campo para investigar el dinero y el equilibrio general está abierto.

Por otro lado, los monetaristas y los nuevos clásicos aceptan la neutralidad del dinero en el corto y el largo plazo, pero advierten que en el corto plazo se puede violar si los agentes forman sus expectativas de manera adaptativa y cometen errores (monetaristas). Incluso con expectativas racionales, los agentes pueden ser enga ados por la autoridad monetaria y confundir los cambios absolutos con cambios relativos en el nivel de precios, y así la política monetaria tiene efectos reales (nuevos clásicos).

La discusión de la inconsistencia dinámica y su solución permite abordar estas deficiencias. Para las dos escuelas, el seguimiento de reglas monetarias soluciona el problema y evita que la política monetaria actúe como factor desestabilizador. Así mismo, la delegación del control monetario a una institución independiente del control gubernamental puede ser otra forma de eliminar la inconsistencia.

En el otro extremo se encuentran los keynesianos, poskeynesianos, nuevos keynesianos y austriacos, quienes acuden a la incertidumbre, el riesgo, las rigideces y los fallos de coordinación que presentan los mercados, para argumentar que el dinero sí tiene efectos reales en la economía.

En estos autores la neutralidad del dinero es un concepto teórico que se verifica bajo supuestos altamente restrictivos. Por lo tanto, en el ámbito de distintas teorías la violación de alguno de los supuestos, que de acuerdo a Patinkin garantizan la neutralidad del dinero, lleva a conclusiones de no neutralidad en el corto plazo, un resultado aceptado por la mayoría de las corrientes teóricas.

Las hipótesis que garantizan la neutralidad del dinero pueden se buenas guías de ejercicios para analizar si en un modelo económico el dinero es neutral o no; también puede ser útil para explicar el mecanismo de transmisión de la política monetaria, lo que permite ver con claridad las relaciones que se establecen en un sistema económico y evaluar los efectos directose 
La neutralidad del dinero y la dicotomía clásica en la ciencia económica

indirectos de las variaciones monetarias. Por otro lado, las hipótesis que garantizan la neutralidad del dinero tienen sentido únicamente si se considera, como lo hace Patinkin, que la oferta monetaria es exógena; si se plantea un modelo con dinero interno los resultados serán distintos a los obtenidos por este autor. Al respecto, Hahn (1982) señala que la discusión en relación al problema teórico de la neutralidad del dinero es un campo abierto a una investigación teórica a fondo.

La consideración de la neutralidad del dinero tiene consecuencias teóricas y empíricas, en la medida en que discutir el papel del dinero implica discutir la política monetaria y el papel del Banco Central y del gobierno en la actualidad. La tendencia actual es tener una política monetaria independiente del gobierno, pero bajo mandatos claros constituidos en reglas. Estas últimas se diseñan bajo el supuesto de ofrecer transparencia ante la opinión pública y clarificar bajo qué condiciones el Banco Central va a intervenir en el mercado. Así mismo, se considera la posibilidad de actuar bajo choques externos, pero con la condición de que la política monetaria no sea utilizada en favor de un gobierno, para evitar el resurgimiento de la inconsistencia dinámica.

Desde el lado de la crítica a este concepto, una de las consecuencias teóricas y empírica relacionados con el dinero es lo concerniente a la tasa de interés. En el libro tercero de El Capital se analiza el desdoblamiento de la ganancia en interés y ganancia de empresa. ¿Cómo opera esta partición? Contrariamente a la ganancia o al salario, "la cuota media de interés vigente en un país ... no puede determinarse en modo alguno por ninguna ley". Es decir, el interés varía entre ciertos límites: puede reducirse a muy poco, pero no puede exceder al total de la ganancia porque el interés es un ingreso derivado, "una rúbrica especial con que se denomina una parte de la ganancia que el capital activo, en vez de embolsarse, tiene que ceder al propietario del capital". El interés no es el precio del capital, que estaría determinado por el valor de una mercancía particular como puede ser el salario para la fuerza del trabajo. Esta diferencia no es solamente cuantitativa, conduce a una distinción cualitativa entre capital financiero y capital industrial, en el sentido de que las dos categorías aparecen como obedeciendo a principios diferentes.

Estamos ante una representación fetichizada del mundo de la producción capitalista, donde el capital produce un ingreso independientemente de la explotación de la fuerza de trabajo: "Para la economía vulgar, que pretende presentar el capital como fuente independiente de valor, de creación de valor, esta forma es, naturalmente, un magnífico hallazgo, la forma en que ya no es posible identificar la fuente de la ganancia y en que el resultado del proceso capitalista de producción-desglosado del proceso mismo- cobra existencia independiente".

Marx vuelve en muchas oportunidades sobre esta ilusión de una puesta en valor aparentemente separada del proceso de producción: "El hecho de que el capital prestado arroje un interés, ya se 
emplee realmente como capital o no -aunque sólo se preste para fines de consumo-, refuerza la idea de la sustantividad de esta forma de capital . También insiste sobre el hecho de que, a la vez, se trata de una representación popular, adecuada para la reificación de las relaciones de producción: "... en la concepción vulgar de la gente se considera el capital-dinero, el capital a interés, como el verdadero capital, como el capital por excelencia".

El abordaje sustractivo (el interés como punción sobre la ganancia) se opone diametralmente, entonces, al de la economía dominante que trataba la repartición del ingreso según una lógica aditiva. En la visión apologética de esta corriente de la economía, la sociedad es un mercado generalizado donde cada uno llega con sus dotaciones para ofrecer sus servicios en el mercado bajo la forma de factores de producción. Algunos ofrecen su trabajo, otros la tierra, otros el capital. Esta teoría, por supuesto, no dice nada sobre el hada madrina que ha atribuido a cada agente sus dotaciones iniciales. Pero la intención es clara: el ingreso nacional está constituido por el agregado de ingresos de diferentes factores de producción según un proceso que tiende a ser armónico. La explotación desaparece porque cada uno de los factores es remunerado según su propia productividad. Este tipo de esquema tiene sus ventajas pero también sus dificultades. Parte de la identificación, de la cual la economía vulgar no se ha desprendido jamás, entre la ganancia y el interés. Para esta corriente de la economía teórica estas dos nociones son, en efecto, indisociables.

Estamos ante una trampa ideológica que Marx señala, al mostrar cómo el capital portador de interés realiza la quintaesencia de la visión burguesa del mundo, porque esta capacidad de hacer del dinero aparece como una propiedad consustancial a este factor de producción. Pero esta solución presenta muchas dificultades a las que es necesario prestar atención. Por ejemplo, generaciones enteras de estudiantes de economía aprenden que el productor maximiza su ganancia . Pero ¿̇cómo se calcula la ganancia?. Es la diferencia entre el precio de producción y el costo de los medios de producción, por lo tanto los salarios, pero también el costo de uso del capital. Este último concepto, relativamente reciente, resume en sí mismo las dificultades de la operación porque depende, a la vez, del precio de las máquinas y de las tasas de interés. Pero si las máquinas y los intereses han sido pagados ¿̇cuál es la ganancia que se maximiza?. La cuestión aún más interesante es que esta ganancia una vez maximizada es nula y, si no lo es, tiende hacia el infinito y la teoría neoclásica de la distribución se derrumba porque los ingresos devienen superiores a la remuneración de cada uno de los factores. La única manera de tratar esta dificultad para la economía dominante es recortarla en pedazos y darle respuestas diferentes según las regiones a explorar, sin jamás asegurar una coherencia de conjunto que sólo podría ser dada por una teoría del valor de la cual no dispone. Para resolver estas dificultades, que conducen a la discusión de Marx, la teoría dominante oscila entre dos posiciones incompatibles. La primera consiste en asimilar el interés a la ganancia y el capital tomado a préstamo al capital involucrado, pero dejando sin explicar la existencia misma de la ganancia de empresa. La segunda consiste en distinguir los dos pero prohibiendo la producción de una teoría unificada del 
La neutralidad del dinero y la dicotomía clásica en la ciencia económica

capital. Toda la historia de la teoría económica burguesa es la de un vaivén entre estas dos posiciones contradictorias, como lo ha mostrado un artículo reciente que examina desde este punto de vista los manuales de economía (Naples et Aslanbeigui, 1996).

\section{BIBLIOGRAFÍA}

Archibald, G.C. y R.G. Lipsey (1958). "Monetary and value theory. A critique ofLange and Patinkin" en Review of Economic Studies, vol. 26.

Ball, L.; N. G. Mankiw y D. Romer. 1988. "The New Keynesian Economics and the Outputinflation Tradeoff", Brookings Papers on Economic Activity 1, pp. 1-65. Reimpreso en N. G. Mankiw y D. Romer, eds., 1991, capítulo 6, pp. 147-211.

Barro, R. . 1989. "The Ricardian Approach to Budget Deficits", Reflections on the Development of Modern Macroeconomics 3, pp. 37-54. Reimpreso en B. Snowdon y H. R. Vane, eds., 1997a, capitulo 13, pp. 314-333.

Barro, R. . 1990. Macroeconomic Policy, Harvard University Press.

Barro, R. . y D. B. Gordon. 1983. "Rules, Discretion and Reputation in a Model of Monetary Policy", Journal of Monetary Economics 12, pp. 101-121.

Benetti, C. (1990). Moneda y teoría del valor, México: UAM/FCE.

Blanchard, O. .y S. Fischer. 1990. Lecture on Macroeconomics, MIT Press.

Blaug, M. (1962). Teoría económica en retrospección, México: FCE, 1985.

Brian, S. Howard, V. y P. Wynarczyk (1994). A modern guide to macroeconomics, Breat Britain:

Edward Elgar Publishing Company.

Cagan, P. 1969. "The Non-neutrality of Money in the Long Run", Journal of Money, Credit and Banking 1, 2, pp. 207-227.

Fischer, S. 1977. "Long-term Contracts, Rational Expectations and the Optimal Money Supply Rule", Journal of Political Economy 85, 1. Reimpreso en N. G. Mankiw y D. Romer, eds., 1991, capítulo 7, pp. 215-231.

Friedman, M. 1968. "The Role of Monetary Policy", American Economic Review 58, 1, pp. 1-17.

Reimpreso en B. Snowdon y H. R. Vane, eds., 1997a, capítulo 7, pp. 164- 179.

González, . I. 1999. "Dicotomía y neutralidad", notas de clase de Macroeconomía Avanzada, Universidad Nacional de Colombia.

González, . I. y A. Pecha. 1995. "La dinámica en economía. Los enfoques de Hicks y Samuelson", Cuadernos de Economía 23, pp. 15-65, Universidad Nacional de Colombia.

Hayek, F.A. (1931). Prices and production, Great Britain: University of London.

Keynes, . M. 1936. Teoría general de la ocupación, el interés y el dinero, Fondo de Cultura Económica. 
Kydland, F. E. y E. C. Prescott. 1977. "Rules Rather than Discretion: The Inconsistency of Optimal Plans", Journal of Political Economy 85, pp. 473-491.

Laidler, D. 1986. "The New-Classical Contribution to Macroeconomics", Banca Nazionale Del Lavoro Quarterly Review, pp. 27-55, marzo. Reimpreso en B. Snowdon y H. R. Vane, eds., 1997a, capítulo 14, pp. 334-358.

Lucas, R. E. y T. Sargent. 1978. "After Keynesian Macroeconomics", After the Phillips Curve: Persistence of High Inflation and High Unemployment, pp. 49-72, Federal Reserve Bank of Boston. Reimpreso en B. Snowdon y H. R. Vane, eds., 1997a, capítulo 11, pp. 270-294.

Mankiw, N. G. 1985. Small Menu Costs and Large Business Cycles: A Macroeconomic Model of Monopoly, N. G. Mankiw y D. Romer, eds., New Keynesian Economics 1, capítulo 1, pp. 29-42, MIT Press, 1991.

Mankiw, N. G. 1990. "A Quick Refresher Course in Macroeconomics", Journal of Economic Literature 28, 4, pp. 1645-1660.

Mankiw, N. G. 2000. "The Inexorable and Mysterious Trade-off between Inflation and Unemployment", NBER Working Paper Series 7884.

Mankiw, N. G. y D. Romer, eds. 1991. New Keynesian Economics 1, MIT Press.

Marx Karl. El Capital. Editorial Fondo de Cultura Económica. Tercera Edición. México. 1978.

Marx Kart. Contribución a la Crítica de la Economía Política. Ediciones Cartago. Buenos Aires. Argentina 1973.

McCallum, B. T. 1989. Monetary Theory and Policy, Macmillan Publishing Company. Minford, P. 1997. "Macroeconomics: Before and After Rational Expectations", B. Snowdon y H. R. Vane, eds., Reflections on the Development of Modern Macroeconomics, capítulo 5, pp. 103127, Edward Elgar Publishing.

Modigliani, F. (1944) "Liquidity preference and the theory of interest and money"en Econometrica, vol. 12, 1944, pp. 45-88. Reprinted in The collected papers ofFranco Modigliani: essays in macroeconomics, vol. 1, MIT Cambridge: Press, 1980.

Patinkin, D. (1989). Money, interest and prices, USA: Massachusetts Institute ofTechnology.

Romer, D. 2002. Advanced Macroeconomics, McGraw-Hill, 2 da edición.

Sargent, T. 1987. Macroeconomic Theory, Academic Press, 2da edición.

Snowdon, B. y H. R. Vane, eds. 1997a. A Macroeconomics Reader, Routledge.

Snowdon, B. y H. R. Vane, eds. 1997b. Reflections on the Development of Modern Macroeconomics, Edward Elgar Publishing.

Snowdon, B.; H. R. Vane y P. Wynarczyk. 1994. A Modern Guide to Macroeconomics: An Introduction to Competing Schools of Thought, Edward Elgar Publishing.

Tobin, . (1965). "Money and economic growth" en Econometrica (26), pp. 671-684.

Walsh, C. 2003. Monetary Theory and Policy, MIT Press, 2da edición. 


\section{NOTAS REFERENCIALES}

${ }^{1}$ Dichas escuelas son presentadas, siguiendo a Snowdon, Vane y Wynarczyk (1994).

2 En la Economía "clásica" Snowdon, Vane y Wynarczyk (1994), consideran que las desviaciones del equilibrio son temporales y el mismo sistema retorna al equilibrio.

${ }^{3}$ Para una forma de resolver este tipo de modelos macroeconómicos, ver el capítulo 1 de Sargent (1987) y el capítulo 5 de McCallum (1989).

${ }^{4}$ Para Sargent $(1987,47)$ "obviamente neutralidad y dicotomía son conceptos distintos. Un sistema neutral no necesariamente dicotomiza, mientras que un sistema en el que el 'dinero es un velo' no tiene por qué satisfacer nuestra definición de sistema neutral. Sin embargo, se considera que la distinción no es tan clara en el sentido en que ambos conceptos conducen a definiciones similares que pueden usarse sin ninguna distinción en términos de este artículo. Ver también González (1999).

${ }^{5}$ Un concepto que trata la literatura y que está relacionado con la neutralidad, aunque no se trate en este artículo, es el de superneutralidad. Blanchard y Fischer (1990) la definen como sigue: "se dice que la moneda es neutral si los cambios en la cantidad nominal no tienen efectos en el equilibrio real. Se dice que es superneutral si los cambios en el crecimiento de la cantidad de moneda no tienen efectos en el equilibrio real" (Blanchard y Fischer, 1990, 207).

${ }^{6}$ Aunque reconocen que puede no presentarse la dicotomía clásica, como se verá más adelante.

${ }^{7}$ Con el fin de explotar la curva de Phillips.

${ }^{8}$ Ver el capítulo 6 de Romer (2002) para una exposición detallada.

${ }^{9}$ Esta característica, presentada por Kydland y Prescott (1977), se denomina inconsistencia dinámica. Para una exposición aplicada a la política monetaria, se puede consultar Barro y Gordon (1983) y la sección 10.4 de Romer (2002).

${ }^{10}$ Con sólo anunciar la política desinflacionaria, el Banco Central puede reducir la inflación sin ningún costo, es decir, la razón de sacrificio, que es la pérdida de producto por reducir la inflación, será cero.

${ }^{11}$ En el contexto de inconsistencia dinámica, la autoridad siempre tiene la tentación de explotar el tradeoff entre inflación y desempleo. Para evitar que exista esa tentación, o por lo menos para mitigarla, se han propuesto como solución al problema de inconsistencia dinámica las siguientes acciones: la independencia del Banco Central, como una forma de delegación del control monetario; el equilibrio de castigo, mediante la pérdida de credibilidad de la sociedad frente al Banco Central; la firma de contrato de incentivos, por medio de los cuales se castigue a los banqueros centrales por el incumplimiento de metas; y el que los miembros de la autoridad monetaria se beneficien de la reputación. Ver el capítulo 10 de Romer (2002) para el detalle de las soluciones relacionadas con la delegación y la reputación y Walsh (2003) para las restantes.

${ }^{12}$ Ver Kydland y Prescott (1977) para una aplicación general, y Barro y Gordon (1983) para una aplicación a la política monetaria.

${ }^{13}$ Ver el capítulo 6 de Snowdon, Vane y Wynarczyk (1994).

${ }^{14}$ Citado en Snowdon, Vane y Wynarczyk (1994, 254).

${ }^{15}$ Citado por Snowdon, Vane y Wynarczyk $(1994,4)$.

${ }^{16}$ Ver los capítulos 2 y 3 de Snowdon, Vane y Wynarczyk (1994).

${ }^{17}$ Aunque las políticas fiscal y monetaria están identificadas con políticas de impulso a la demanda agregada, para los keynesianos la más efectiva de ellas es la fiscal. La monetaria tiende a ser irrelevante por la posibilidad de que se caiga en la trampa de liquidez. Sin embargo, Tobin (1987) citado en Snowdon, Vane y Wynarczyk (1994) muestra que los factores monetarios también son importantes para la economía keynesiana.

${ }^{18}$ Por ejemplo, generar un superávit fiscal.

${ }^{19}$ La política económica, en el contexto keynesiano, debería ser contra cíclica. 
${ }^{20}$ Ver Mankiw y Romer (1991) para los desarrollos de los nuevos keynesianos, y el capítulo 7 de Snowdon, Vane y Wynarczyk (1994) para analizar sus proposiciones fundamentales.

${ }^{21}$ Por la existencia de rigideces nominales y reales. Un ejemplo son los costos de menú (ver Mankiw, 1985 y Romer, 2002, capítulo 6).

${ }^{22}$ Ver Snowdon, Vane y Wynarczyk (1994), capítulo 8, sección 8.9.4.

23 (Ibíd., 1994), capítulos 1 y 4.

${ }^{24}$ El rezago endógeno es el tiempo transcurrido entre el momento en que se decide ejecutar la política y el momento en que se ejecuta. El exógeno es el tiempo que transcurre entre el momento en que se ejecuta y sus efectos. Se dice que la política fiscal tiene un gran rezago endógeno y un pequeño rezago exógeno, mientras la política monetaria tiene un rezago endógeno pequeño y uno exógeno muy grande que varía de país a país.

${ }^{26}$ C. Marx, Elementos Fundamentales para la Crítica de la Economía Política. Editorisal Cartago. Vol I, pp 65-66, 68-69, 93. Buenos Aires. Argentina. 1974.

${ }^{27}$ C. Marx, El Capital, Editorial Fondo de Cultura Económica, t. III, p. 567. México DF. 1978.

${ }^{28}$ C. Marx, El Capital, cit, t.. III, pp. 472-73.

${ }^{29}$ C. Marx, Elementos Fundamentales ..., cit, vol. I, pp, 61-65, 76-79. Contribución a la Crítica de la Economía Política, cit., pp. 89-92.

${ }^{30}$ C. Marx, Elementos Fundamentales ..., cit., vol. I, p. 93.

${ }^{31}$ Rudolf Hilferding, El Capital Financiero. Ed. Tecnos SA, Barcelona, España, 1982, p. 39.

32 Kart Kautsky, "Gold, Papier und Ware", en Die Neue Zeit, vol. 31/31, no. 24, p. 837.

33 V.I. Lenin, Obras Completas. Ed. Akal, t. XLIII (Cuadernos sobre el imperialismo), Madrid, España, 1981 p. 325 ,

34 Suzanne de Brunhoff. La Oferta de la Moneda. Ed. Tiempo Contemporáneo, Buenos Aires, 1975, pp. 12832.

35 C. Marx, Elementos Fundamentales ..,, cit., vol. I, pp. 64-65.

36 C. Marx, Elementos Fundamentales ... cit., vol. I, p. 120.

37 C. Marx, Contribución a la Crítica de la Economía Política ..., cit, p. 73. 